Braz J Med Biol Res, September 2011, Volume 44(9) 864-870

doi: 10.1590/S0100-879X2011007500100

Strength and power training did not modify cardiovascular responses to aerobic exercise in elderly subjects

H. Kanegusuku, A.C.C. Queiroz, M.R. Chehuen, L.A.R. Costa, L.F. Wallerstein, M.T. Mello, C. Ugrinowitsch and C.L.M. Forjaz

The Brazilian Journal of Medical and Biological Research is partially financed by

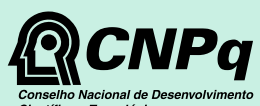

Ministério

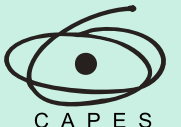

Ministério

da Educação

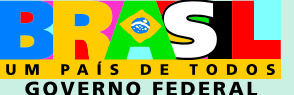

DTAPESP

Institutional Sponsors

seifo 0
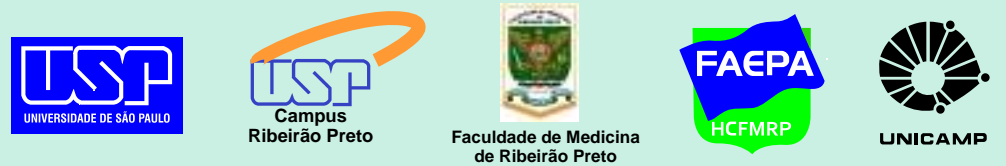

Ф SHIMADZU

Explore High - Performance MS

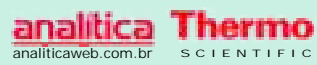




\title{
Strength and power training did not modify cardiovascular responses to aerobic exercise in elderly subjects
}

\author{
H. Kanegusuku ${ }^{1}$, A.C.C. Queiroz ${ }^{1}$, M.R. Chehuen ${ }^{1}$, L.A.R. Costa ${ }^{1}$, \\ L.F. Wallerstein ${ }^{2}$, M.T. Mello ${ }^{3}$, C. Ugrinowitsch ${ }^{2}$ and C.L.M. Forjaz ${ }^{1}$ \\ ${ }^{1}$ Laboratório de Hemodinâmica da Atividade Motora, Escola de Educação Física e Esporte, \\ Universidade de São Paulo, São Paulo, SP, Brasil \\ ${ }^{2}$ Laboratório de Adaptação Neuromuscular ao Treinamento de Força, Escola de Educação Física e Esporte, \\ Universidade de São Paulo, São Paulo, SP, Brasil \\ ${ }^{3}$ Centro de Estudos em Psicobiologia e Exercício, Universidade Federal de São Paulo, São Paulo, SP, Brasil
}

\begin{abstract}
Resistance training increases muscle strength in older adults, decreasing the effort necessary for executing physical tasks, and reducing cardiovascular load during exercise. This hypothesis has been confirmed during strength-based activities, but not during aerobic-based activities. This study determined whether different resistance training regimens, strength training (ST, constant movement velocity) or power training (PT, concentric phase performed as fast as possible) can blunt the increase in cardiovascular load during an aerobic stimulus. Older adults $(63.9 \pm 0.7$ years) were randomly allocated to: control $(\mathrm{N}=11)$, ST ( $N=13$, twice a week, 70-90\% 1-RM) and PT ( $N=15$, twice a week, 30-50\% 1-RM) groups. Before and after 16 weeks, oxygen uptake $\left(\mathrm{VO}_{2}\right)$, systolic blood pressure (SBP), heart rate (HR), and rate pressure product (RPP) were measured during a maximal treadmill test. Resting SBP and RPP were similarly reduced in all groups (combined data $=-5.7 \pm 1.2$ and $-5.0 \pm$ $1.7 \%$, respectively, $\mathrm{P}<0.05$ ). Maximal SBP, HR and RPP did not change. The increase in measured $\mathrm{VO}_{2}, \mathrm{HR}$ and RPP for the increment in estimated $\mathrm{VO}_{2}$ (absolute load) decreased similarly in all groups (combined data $=-9.1 \pm 2.6,-14.1 \pm 3.9,-14.2$ $\pm 3.0 \%$, respectively, $\mathrm{P}<0.05$ ), while the increments in the cardiovascular variables for the increase in measured $\mathrm{VO}_{2}$ did not change. In elderly subjects, ST and PT did not blunt submaximal or maximal HR, SBP and RPP increases during the maximal exercise test, showing that they did not reduce cardiovascular stress during aerobic tasks.
\end{abstract}

Key words: Aging; Resistance training; Maximal exercise test; Aerobic exercise

\section{Introduction}

The aging process is associated with many alterations in muscle structure and function, which lead to a reduction in muscle strength, mass, and power (1-3). Since these factors are very important for the performance of many daily life activities, these alterations may reduce the quality of life of older individuals (4).

In order to prevent or reverse those changes due to aging, different resistance training regimens have been recommended for elderly subjects (5-7). When resistance training is performed with high loads and low movement velocity, it is called strength training (ST). The main effects of ST are improvements in muscle strength and mass, and this kind of training is recommended for reducing or reversing sarcopenia in older adults (8). On the other hand, when performed with low loads and high movement velocity, resistance training is called power training (PT) because it mainly increases muscle power production. $\mathrm{PT}$ is recommended for older subjects to improve muscle functionality during daily life activities that require rapid movements $(9,10)$.

In addition to affecting the neuromuscular system, resistance training may also promote benefits for the cardiovascular system by reducing resting heart rate (HR) (11) and/or blood pressure (BP) in normotensive elderly subjects (12-15). In addition, the increase in muscle strength and power produced by resistance training may reduce

Correspondence: C.L.M. Forjaz, Av. Prof. Mello Moraes, 65, 05508-030 São Paulo, SP, Brasil. Fax: +55-11-3813-5921.

E-mail: cforjaz@usp.br

Presented at the XV Simpósio Brasileiro de Fisiologia Cardiovascular, São Paulo, SP, Brazil, February 2-5, 2011.

Received February 7, 2011. Accepted July 27, 2011. Available online August 12, 2011. Published September 16, 2011. 
the effort necessary to perform physical activities (13). As a consequence, the cardiovascular load may be reduced during these tasks. Accordingly, McCartney et al. (16) showed that elderly subjects presented a lower increase in systolic BP (SBP) during the execution of a resistance exercise with the same absolute workload after a period of resistance training. The attenuated increase in BP may be of clinical relevance for elderly subjects, helping to reduce cardiovascular risk during daily activities that require muscle strength production, such as lifting, carrying or pushing heavy objects.

Nevertheless, many daily life activities performed by elderly subjects, such as walking, sweeping the house, and washing dishes, have aerobic and not strength characteristics. Thus, it seems important to evaluate the effects of resistance training on the cardiovascular responses to an aerobic stimulus. It is known that the increase in SBP during aerobic exercise is a good predictor of cardiovascular risk, independently of the resting SBP (17). If resistance training does lessen the effort necessary to perform an aerobic exercise, the health risk will be lower for elderly subjects due to the blunted cardiovascular response during this exercise. This cardiovascular benefit is interesting because aging is associated with important cardiovascular alterations, such as increases in systemic vascular resistance, cardiovascular sympathetic modulation, HR, and BP combined with decreases in cardiac parasympathetic modulation and baroreflex sensitivity (18-23). All of these changes increase the probability of developing cardiovascular diseases, which are the most important causes of death in Brazil (24).

Therefore, this study was designed to determine whether different resistance training regimens (ST and PT) could reduce the cardiovascular load during aerobic exercise. $A$ maximal exercise test was chosen because it permits the evaluation of the cardiovascular load at both submaximal and maximal intensities. The hypothesis was that both resistance training regimens would increase muscle strength, improve the efficiency of exercise, and reduce the cardiovascular load during exercise.

\section{Material and Methods}

\section{Study population}

Thirty-nine normotensive older adults (60 to 80 years) volunteered for this study. The study was approved by the Ethics Committee of Escola de Educação Física e Esporte, Universidade de São Paulo and subjects were informed about the risks and benefits involved in participating in the study and gave written informed consent to participate. The study was registered in the ClinicalTrials.gov system under registration No. NCT01113203.

Subjects who presented high BP levels and/or cardiovascular abnormalities in a resting or exercise ECG were excluded. None of the participants had a previous diagnosis of cardiovascular or musculoskeletal diseases. Moreover, none of them smoked, took medications that could affect the cardiovascular responses to exercise, or participated in any exercise program.

The volunteers were randomly allocated to the ST $(\mathrm{N}=$ 13), PT ( $=15)$, and control $(C, N=11)$ groups. Subjects in the $\mathrm{C}$ group underwent all the evaluations but did not take part in any training program. All volunteers were asked not to change their lifestyle during the 16 weeks of the study period.

\section{Preliminary evaluation}

Subjects' auscultatory blood pressure was measured three times after $5 \mathrm{~min}$ of seated rest on two separate occasions. The first and the fifth Korotkoff's sounds were considered for SBP and diastolic BP (DBP) determinations, respectively. The mean value of these measurements was calculated to determine subjects' $\mathrm{BP}(25)$. Subjects were excluded if they had SBP and/or DBP $\geq 140 / 90 \mathrm{mmHg}$ (25).

To assess the effectiveness of ST and PT in promoting muscular adaptations, muscle strength was measured by the 1 repetition maximum (1-RM) test described by Brown and Weir (26) in the leg press and chest press exercises (Technogym, Italy).

\section{Experimental procedures}

All subjects were evaluated during a progressive maximal cardiopulmonary exercise test, before and 16 weeks after the interventions. In both evaluations, subjects were instructed to eat a light meal $2 \mathrm{~h}$ before the test, to avoid coffee, tea, coke, alcohol, or other central nervous stimulant substances on the test day, and not to perform physical exercise during the $48 \mathrm{~h}$ preceding the test.

The test was conducted on a treadmill (Inbrasport, ATL, Brazil) using an individualized Balke's modified protocol chosen in order to achieve fatigue between 8 and 12 min of testing. Thus, according to the subjects' fitness level, which was estimated by an exercise test specialist based on the physical activity reported by the subjects, the treadmill speed was set at $3.0,3.4$, or $4.0 \mathrm{mph}$, and the inclination was increased $2 \%$ every minute until the subjects were unable to continue (27). The same protocol was repeated for each subject before and after the interventions. The estimated metabolic cost for each exercise workload was calculated based on the American College of Sports Medicine's (ACSM) equation: estimated $\mathrm{VO}_{2}(\mathrm{~L} / \mathrm{min})=[($ speed $\times 0.1)+($ speed $\mathrm{x}$ inclination $\times 1.8)+3.5] \times$ weight / 1000. HR was continuously monitored with an ECG (CardioPerfect MD, BC Delft, The Netherlands), and auscultatory BP was measured every 2 min. BP measurements before and after training were made by the same technician who was blind to the subject's group. $\mathrm{VO}_{2}$ was continuously assessed with a metabolic cart (Medical Graphics Corporation, CPX/D, USA), and values were averaged over 1-min intervals for further analysis. The ratepressure product (RPP), an indirect index of cardiac workload, was calculated by the product of HR and SBP. 


\section{Exercise training programs}

Both training programs (ST and PT) consisted of 2 exercise sessions per week for 16 weeks. Training sessions were conducted and supervised by an experienced strength coach. Each session consisted of a 10-min warm-up period on a treadmill, followed by the execution of 7 resistance exercises (horizontal leg press, bilateral knee flexion, unilateral hip extension, plantar flexion in the horizontal leg press, chest press, lat pull down, and upright) on isoinertial machines (Technogym). In both training protocols, an interval of at least 3 min was allowed between sets and exercises. To assure the progression of training, the number of sets and the intensity of exercises changed throughout the experimental period for both training groups. These changes were different between the ST and the PT protocols, and are shown in Table 1. In addition, in the ST protocol, subjects were instructed to perform the exercises at a comfortable and constant movement speed (i.e., $2 \mathrm{~s}$ for the concentric phase and 2 $s$ for the eccentric phase) throughout the training period. In contrast, in the PT group, subjects were instructed to perform the concentric phase as fast as possible and to keep a comfortable speed in the eccentric phase. The ST and PT protocols employed in the present study were designed on the basis of the specific recommendations for each training regimen in order to best potentiate the gain in strength and power, and they were not elaborated to produce a similar physical overload. When comparing the two, it is possible to observe that they present a similar volume (number of sets multiplied by number of repetitions in each set), and their main differences have to do with the intensity of the exercises and the velocity of movement.

\section{Data analysis}

Resting data were measured on the treadmill during the last $30 \mathrm{~s}$ before starting the exercise test. Maximal data were defined as the greatest values achieved during the test.

To evaluate the physiological responses to submaximal exercise, linear regressions were calculated for: 1) measured and estimated $\mathrm{VO}_{2}$ to assess the possible effect of training on the metabolic efficiency of exercise; 2) cardiovascular parameters and estimated $\mathrm{VO}_{2}$ to assess a possible effect of training on similar workload exercises, and 3) cardiovascular parameters and measured $\mathrm{VO}_{2}$ to assess the possible effect of training on a stimulus of similar aerobic demand. These regressions were calculated on the basis of the values measured at rest before the test and during it, excluding the maximal values. The slopes of these regressions were considered for analysis. As an example, the individual and mean regressions, calculated before and after the intervention in one of the groups, are shown in Figure 1.

\section{Statistical analyses}

The normal distribution of the data was checked using the Shapiro-Wilks test (SPSS for Windows, USA). The preintervention values of the two groups were compared by one-way ANOVA.

The effects of training on the experimental groups were assessed by two-way ANOVA for repeated measures (Statsoft, Statistica for Windows, USA), with group (ST, PT, and $\mathrm{C}$ ) as a between-subject factor, and time (pre- and postintervention) as a within-subject factor. In the case of significant $F$ values, the Newman-Keuls post hoc test was used for multiple comparison purposes. $\mathrm{P}<0.05$ was accepted as significant, and data are reported as means \pm SEM.

Table 1. Strength (ST) and power training (PT) protocols over the 16-week training period.

\begin{tabular}{lcccccccc}
\hline & \multicolumn{3}{c}{ ST } & & \multicolumn{3}{c}{ PT } \\
\cline { 2 - 4 } \cline { 6 - 8 } & Sets & Repetitions & $\begin{array}{c}\text { Intensity } \\
(\%)\end{array}$ & & Sets & Repetitions & $\begin{array}{c}\text { Intensity } \\
(\%)\end{array}$ \\
\hline Weeks 1-2 & 2 & 10 & 70 & & 3 & 7 & 30 \\
Weeks 3-4 & 2 & 10 & 75 & & 3 & 7 & 35 \\
Weeks 5-8 & $2+1$ & $10+8$ & $75+80$ & & $3+1$ & $7+6$ & $30+40$ \\
Weeks 9-12 & 3 & 8 & 80 & & 4 & 6 & 40 \\
Weeks 13-14 & $2+2$ & $8+6$ & $80+85$ & $2+2$ & $6+4$ & $40+45$ \\
Weeks 15-16 & $2+2$ & $6+4$ & $85+90$ & $2+2$ & $6+4$ & $45+50$ \\
\hline
\end{tabular}
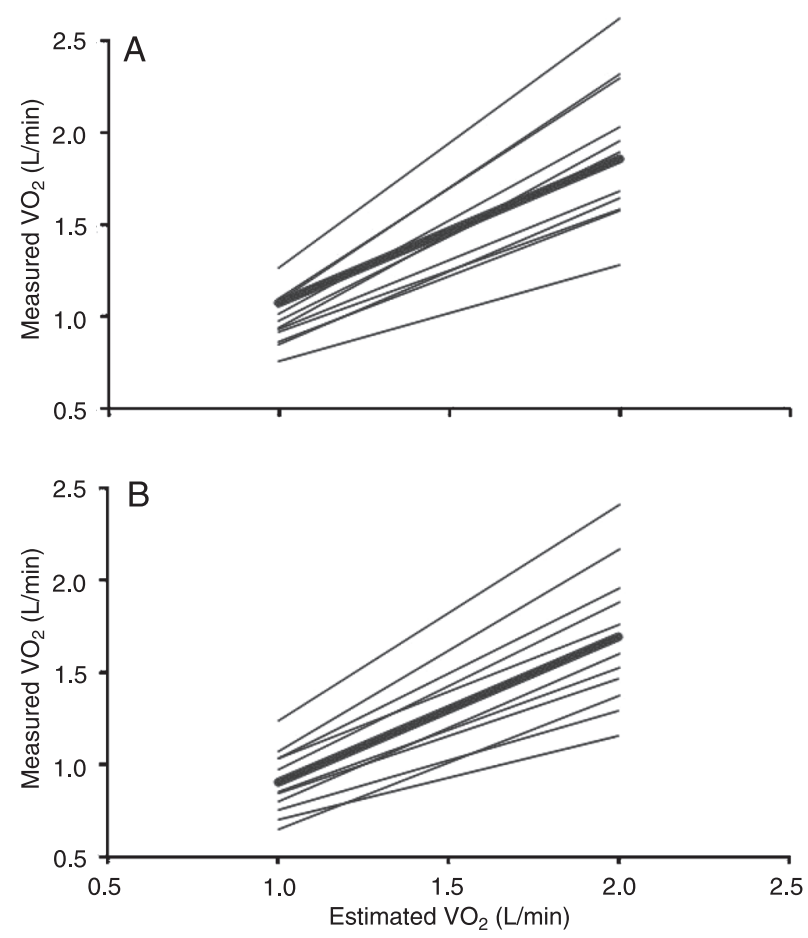

Figure 1. Individual (thin lines) and mean (thick line) regression lines obtained for the measured and estimated oxygen uptake $\left(\mathrm{VO}_{2}\right)$ before $($ Panel $A)$ and after $($ Panel $B) 16$ weeks of intervention in the strength training (ST) group. 


\section{Results}

Pre-intervention physical, hemodynamic, and muscular characteristics were similar for the experimental groups (Table 2). During the study, some of the volunteers were taking medications that did not have a specific effect on cardiovascular responses to exercise. Three subjects from each experimental group were receiving oral hypoglycemic drugs, and 3 subjects (2 from group $\mathrm{C}$, and 1 from group PT) were receiving statins.

Adherence to exercise training was greater than $95 \%$ in both training groups. Weight and body mass index did not change in any group. When compared before and after the interventions, muscle strength increased significantly and similarly in the ST and PT groups (leg press $=115.3 \pm 14.1$ vs $164.3 \pm 18.2$ and $99.9 \pm 10.0$ vs $133.5 \pm 13.2 \mathrm{~kg}$, and chest press $=32.7 \pm 3.4$ vs $42.7 \pm 4.0$ and $28.9 \pm 2.6$ vs $36.6 \pm 3.1 \mathrm{~kg}$, respectively, $\mathrm{P}$ $<0.05$ ), but did not change in the $C$ group (leg press $=90.8 \pm$ 12.3 vs $88.5 \pm 11.3 \mathrm{~kg}$, and chest press $=29.5 \pm 3.0$ vs 30.3 $\pm 3.0 \mathrm{~kg}, \mathrm{P}>0.05)$.

All but 2 maximal tests lasted between 8 and $12 \mathrm{~min}$. At the end of the maximal test, all subjects except 1 reached at least $90 \%$ of their age-predicted maximal HR or a respiratory quotient $\geq 1.05$. The percent of age-predicted maximal HR achieved by the subjects in the tests were similar for the groups before the interventions, and did not change after the experimental period
$(\mathrm{C}=102 \pm 2$ vs $100 \pm 2 ; \mathrm{ST}=104 \pm 2$ vs $105 \pm 3 ; \mathrm{PT}=105 \pm$ 2 vs $106 \pm 2 \%$ of age-predicted maximal HR, P > 0.05).

The maximal exercise time, as well as the estimated $\mathrm{VO}_{2}$ peak and the measured $\mathrm{VO}_{2}$ peak, were similar for the groups at the beginning of the study and increased significantly and similarly after the interventions in all groups (Table 3).

Table 2. Characteristics of the subjects allocated to each group at the beginning of the study.

\begin{tabular}{lccc}
\hline & $\mathrm{C}$ & $\mathrm{ST}$ & $\mathrm{PT}$ \\
\hline $\mathrm{N}$ & 11 & 13 & 15 \\
Male/female & $2 / 9$ & $5 / 8$ & $4 / 11$ \\
Age (years) & $63 \pm 1$ & $63 \pm 1$ & $65 \pm 1$ \\
Weight $(\mathrm{kg})$ & $69.2 \pm 3.3$ & $72.6 \pm 4.8$ & $65.5 \pm 3.3$ \\
BMI $\left(\mathrm{kg} / \mathrm{m}^{2}\right)$ & $27.3 \pm 1.5$ & $27.4 \pm 1.6$ & $26.5 \pm 1.2$ \\
Systolic BP (mmHg) & $124.4 \pm 2.1$ & $123.2 \pm 1.9$ & $120.8 \pm 2.4$ \\
Diastolic BP $(\mathrm{mmHg})$ & $78.3 \pm 1.2$ & $78.7 \pm 1.4$ & $77.9 \pm 1.5$ \\
Mean BP $(\mathrm{mmHg})$ & $93.7 \pm 1.4$ & $93.6 \pm 1.5$ & $92.2 \pm 1.6$ \\
HR (bpm) & $66.6 \pm 2.2$ & $69.6 \pm 1.4$ & $67.8 \pm 1.5$ \\
1-RM leg press $(\mathrm{kg})$ & $90.8 \pm 12.3$ & $115.3 \pm 14.1$ & $99.9 \pm 10.0$ \\
1-RM chest press $(\mathrm{kg})$ & $29.5 \pm 3.0$ & $32.7 \pm 3.4$ & $28.9 \pm 2.6$ \\
\hline
\end{tabular}

Data are reported as means \pm SEM. $C=$ control; $\mathrm{ST}=$ strength training; $\mathrm{PT}=$ power training; $\mathrm{BMI}=$ body mass index, $\mathrm{BP}=$ blood pressure; $\mathrm{HR}=$ heart rate.

Table 3. Variables measured before and during the maximal exercise test, performed before and after the interventions.

\begin{tabular}{|c|c|c|c|c|c|c|}
\hline & \multicolumn{2}{|c|}{ C } & \multicolumn{2}{|c|}{ ST } & \multicolumn{2}{|c|}{ PT } \\
\hline & Pre & Post & Pre & Post & Pre & Post \\
\hline Test time (min) & $9.9 \pm 0.7$ & $10.6 \pm 0.8^{*}$ & $8.1 \pm 0.7$ & $10.1 \pm 0.7^{*}$ & $9.3 \pm 0.5$ & $10.5 \pm 0.6^{*}$ \\
\hline \multicolumn{7}{|l|}{ Metabolic variables } \\
\hline Estimated $\mathrm{VO}_{2}$ peak (L/min) & $2.06 \pm 0.15$ & $2.26 \pm 0.20^{*}$ & $1.83 \pm 0.24$ & $2.17 \pm 0.18^{*}$ & $1.98 \pm 0.17$ & $2.24 \pm 0.13^{*}$ \\
\hline Measured $\mathrm{VO}_{2}$ peak (L/min) & $1.58 \pm 0.08$ & $1.64 \pm 0.09^{*}$ & $1.56 \pm 0.14$ & $1.69 \pm 0.12^{*}$ & $1.49 \pm 0.09$ & $1.67 \pm 0.09^{*}$ \\
\hline \multicolumn{7}{|l|}{$\begin{array}{l}\text { Cardiovascular variables } \\
\text { Pre-exercise rest }\end{array}$} \\
\hline $\mathrm{HR}(\mathrm{bpm})$ & $81 \pm 4$ & $78 \pm 4$ & $83 \pm 5$ & $87 \pm 4$ & $76 \pm 4$ & $77 \pm 3$ \\
\hline $\mathrm{SBP}(\mathrm{mmHg})$ & $127 \pm 4$ & $118 \pm 3^{*}$ & $121 \pm 5$ & $116 \pm 5^{*}$ & $125 \pm 4$ & $117 \pm 4^{*}$ \\
\hline $\mathrm{DBP}(\mathrm{mmHg})$ & $77 \pm 4$ & $73 \pm 3$ & $78 \pm 3$ & $75 \pm 3$ & $75 \pm 3$ & $73 \pm 3$ \\
\hline $\operatorname{RPP}\left(\mathrm{mmHg} \cdot \mathrm{bpm} \cdot 10^{3}\right)$ & $10.1 \pm 0.4$ & $9.3 \pm 0.4^{*}$ & $9.6 \pm 0.8$ & $9.6 \pm 0.7^{*}$ & $9.4 \pm 0.6$ & $8.7 \pm 0.7^{*}$ \\
\hline \multicolumn{7}{|l|}{ Maximal value } \\
\hline $\mathrm{HR}(\mathrm{bpm})$ & $160 \pm 4$ & $156 \pm 4$ & $162 \pm 4$ & $164 \pm 5$ & $163 \pm 3$ & $163 \pm 2$ \\
\hline $\mathrm{SBP}(\mathrm{mmHg})$ & $187 \pm 8$ & $186 \pm 9$ & $196 \pm 6$ & $192 \pm 6$ & $183 \pm 5$ & $177 \pm 5$ \\
\hline $\operatorname{RPP}\left(\mathrm{mmHg} \cdot \mathrm{bpm} \cdot 10^{3}\right)$ & $30.0 \pm 1.2$ & $28.5 \pm 1.6$ & $31.8 \pm 1.3$ & $31.6 \pm 1.3$ & $29.6 \pm 1.1$ & $29.0 \pm 1.1$ \\
\hline \multicolumn{7}{|c|}{ Maximal increase during exercise } \\
\hline$\Delta \mathrm{HR}(\mathrm{bpm})$ & $+80.3 \pm 4.8$ & $+77.9 \pm 4.7$ & $+75.8 \pm 4.9$ & $+75.0 \pm 5.3$ & $+89.3 \pm 3.8$ & $+89.8 \pm 3.6$ \\
\hline$\Delta \mathrm{SBP}(\mathrm{mmHg})$ & $+61.5 \pm 6.8$ & $+66.3 \pm 6.9^{*}$ & $+70.7 \pm 6.5$ & $+78.8 \pm 6.3^{*}$ & $+56.3 \pm 3.8$ & $+62.0 \pm 3.4^{*}$ \\
\hline$\Delta \mathrm{RPP}\left(\mathrm{mmHg} \cdot \mathrm{bpm} \cdot 10^{3}\right)$ & $+4.7 \pm 0.5$ & $+4.6 \pm 0.5$ & $+5.7 \pm 0.6$ & $+6.2 \pm 0.7$ & $+4.8 \pm 0.4$ & $+5.1 \pm 0.4$ \\
\hline
\end{tabular}

Data are reported as means \pm SEM. $C=$ control; $\mathrm{ST}=$ strength training; $\mathrm{PT}=$ power training; $\mathrm{VO}_{2}=$ oxygen uptake; $\mathrm{HR}=$ heart rate; $\mathrm{SBP}=$ systolic blood pressure; $\mathrm{DBP}=$ diastolic blood pressure; $\mathrm{RPP}=$ rate pressure product. ${ }^{*} \mathrm{P}<0.05$ compared to the pre-intervention period (two-way ANOVA for repeated measures). 
Table 4. Linear regression slopes calculated for the variables measured during the maximal exercise test, performed before and after the interventions.

\begin{tabular}{|c|c|c|c|c|c|c|}
\hline & \multicolumn{2}{|c|}{ C } & \multicolumn{2}{|c|}{ ST } & \multicolumn{2}{|c|}{ PT } \\
\hline & Pre & Post & Pre & Post & Pre & Post \\
\hline Measured $\mathrm{VO}_{2} \times$ estimated $\mathrm{VO}_{2}$ & $0.84 \pm 0.10$ & $0.81 \pm 0.10^{*}$ & $0.91 \pm 0.08$ & $0.79 \pm 0.09^{*}$ & $0.82 \pm 0.04$ & $0.73 \pm 0.06^{*}$ \\
\hline $\mathrm{HR} \times$ estimated $\mathrm{VO}_{2}$ & $45.7 \pm 5.7$ & $40.9 \pm 5.8^{*}$ & $54.8 \pm 8.5$ & $39.2 \pm 5.5^{\star}$ & $54.3 \pm 5.4$ & $45.2 \pm 2.8^{*}$ \\
\hline SBP $x$ estimated $\mathrm{VO}_{2}$ & $40.3 \pm 6.7$ & $39.4 \pm 6.7$ & $42.6 \pm 3.9$ & $40.5 \pm 4.4$ & $39.8 \pm 3.6$ & $34.2 \pm 2.1$ \\
\hline $\mathrm{RPP} \times$ estimated $\mathrm{VO}_{2}$ & $11.39 \pm 1.48$ & $10.16 \pm 1.45^{*}$ & $11.93 \pm 1.21$ & $9.56 \pm 0.96^{*}$ & $12.54 \pm 1.00$ & $10.44 \pm 0.65^{*}$ \\
\hline $\mathrm{HR} \times$ measured $\mathrm{VO}_{2}$ & $46.5 \pm 5.0$ & $46.1 \pm 4.8$ & $47.8 \pm 4.7$ & $57.0 \pm 6.1$ & $45.3 \pm 3.6$ & $44.1 \pm 4.0$ \\
\hline $\mathrm{SBP} \times$ measured $\mathrm{VO}_{2}$ & $51.5 \pm 3.1$ & $49.9 \pm 3.4$ & $58.6 \pm 6.3$ & $49.2 \pm 3.9$ & $62.6 \pm 4.6$ & $62.8 \pm 4.2$ \\
\hline $\mathrm{RPP} \times$ measured $\mathrm{VO}_{2}$ & $13.10 \pm 0.85$ & $12.09 \pm 1.00$ & $14.04 \pm 1.01$ & $13.73 \pm 1.05$ & $14.19 \pm 0.96$ & $14.11 \pm 1.11$ \\
\hline
\end{tabular}

Data are reported as means $\pm \mathrm{SEM} . \mathrm{C}=$ control; $\mathrm{ST}=$ strength training; $\mathrm{PT}=$ power training; $\mathrm{VO}_{2}=$ oxygen uptake $(\mathrm{L} / \mathrm{min}) ; \mathrm{HR}=$ heart rate $(\mathrm{bpm}) ; \mathrm{SBP}=$ systolic blood pressure $(\mathrm{mmHg}) ; \mathrm{RPP}=$ rate pressure product $\left(\mathrm{mmHg} \cdot \mathrm{bpm} \cdot 10^{3}\right)$. ${ }^{*} \mathrm{P}<0.05$ compared to the preintervention period (two-way ANOVA for repeated measures).

The resting and maximal values, as well as the maximal increase $(\Delta)$ of HR, SBP and RPP measured during the incremental test for the three groups, before and after the interventions, are shown in Table 3. In the pre-intervention period, all of these variables were similar for the experimental groups. After the intervention, resting SBP and RPP decreased significantly and similarly in all experimental groups, while HR and DBP did not change. Maximal HR, SBP and RPP did not change in the groups. Thus, the maximal increase in SBP $(\triangle \mathrm{SBP})$ during the incremental test was significant and similar from pre- to post-intervention in all groups.

The mean of the linear regression slopes (mean of the slopes) calculated before and after the interventions for each of the three groups, are presented in Table 4. In the pre-intervention period, the means of the slopes of the three groups were similar. After the interventions, the mean of the slopes calculated for measured $\mathrm{VO}_{2}, \mathrm{HR}$, and RPP in regard to the estimated $\mathrm{VO}_{2}$ decreased significantly and similarly in all experimental groups. The means of the slopes of SBP and estimated $\mathrm{VO}_{2}$ did not change in either group. The means of the slopes calculated for all the cardiovascular parameters in regard to the measured $\mathrm{VO}_{2}$ did not change after the interventions in either group.

\section{Discussion}

The main result of the present study was that the ST and PT regimens used did not reduce the cardiovascular load during submaximal and maximal aerobic stimuli.

It is known that high-intensity-low velocity and lowintensity-high velocity resistance training regimens promote important muscle adaptations in the elderly $(8-10,28)$. In the present study, the ST and PT regimens were equally effective in increasing muscle strength (41.0 and 34.9\%, respectively), and the magnitude of increment was similar to the gains reported in other studies (29), supporting that both training regimens improved the muscular function of elderly individuals.

Resting SBP and, consequently, RPP were similarly reduced in all experimental groups, including group $\mathrm{C}$, which shows that this reduction cannot be attributed to either of the training regimens. Results regarding the changes in resting SBP after resistance training are equivocal in the literature. Some previous studies reported a reduction in resting SBP in normotensive elderly people (13-15), while others $(11,30)$ reported results similar to those presented here. In addition, ST did not change resting HR, which is in accordance with some studies $(13,14,31)$, but differs from others that reported a reduction (11) or even an increase in HR (32). To our knowledge, no previous study observed cardiovascular responses in the elderly after PT. The differences between previous studies and the present one may be attributed to the ST protocols employed, mainly differences in the training period, intensity, number of exercises, sets and repetitions, and/or to the absence of a control group.

The reduction in resting SBP observed in all groups might be due to an adaptation to the measurement procedure that decreases the alert response to the measurement process $(33,34)$. Since the decreases were similar among the groups, they did not represent an effect of training.

After the interventions, a similar increase in maximal exercise time and in $\mathrm{VO}_{2}$ peak was detected for all groups, including group $\mathrm{C}$. Thus, this improvement in exercise performance cannot be related to the resistance exercise programs. Instead, when elderly subjects repeat a test, they adapt to it, reducing unnecessary movements, decreasing the metabolic cost for a fixed workload, and improving performance (35). The similar decrease in the linear regression slope between the measured and estimated $\mathrm{VO}_{2}$ supports this hypothesis. Therefore, in contrast to the study's hypothesis, resistance training did not improve 
exercise efficiency.

The absence of any effect of the ST and PT regimens on the measured $\mathrm{VO}_{2}$ peak was expected. It has been widely reported in the literature that resistance training, especially when performed with high intensity, does not increase the $\mathrm{VO}_{2}$ peak (36). In addition, resistance training did not affect the maximal HR and SBP, which had already been observed after the ST (37). However, to our knowledge, no previous study had evaluated this response after PT. Thus, the present study expands the knowledge in this area by showing that, similar to ST, PT also does not affect the maximal cardiovascular response to an aerobic stimulus.

Since the ST and $\mathrm{PT}$ regimens did not influence resting and maximal HR, RPP and SBP values, they also did not affect the rise of these variables during the maximal exercise. Since a huge increase in SBP during a maximal test is a predictor of cardiovascular and non-cardiovascular deaths (17), the present results suggest that resistance training did not reduce this risk in elderly subjects.

In the present study, cardiovascular responses to submaximal exercise were analyzed by the linear regression slope calculated between the cardiovascular parameters, and the estimated and measured $\mathrm{VO}_{2}$. When slopes for the estimated $\mathrm{VO}_{2}$ are analyzed, they represent a comparison for the same absolute workload. Thus, the results showed that the increases in HR and RPP for each increment in workload decreased after the interventions, but as these responses were similar for all groups, they did not represent a training effect. In fact, they reflected the increase in the work efficiency promoted by the adaptation to the test, as previously discussed. When cardiovascular responses to a submaximal exercise were analyzed in regard to the measured $\mathrm{VO}_{2}$, they represented the response to the same aerobic stress. As the increases in HR, SBP and RPP for each min of increase in measured $\mathrm{VO}_{2}$ were similar before and after the interventions in all groups, it is possible to

\section{References}

1. Larsson L. Morphological and functional characteristics of the ageing skeletal muscle in man. A cross-sectional study. Acta Physiol Scand Suppl 1978; 457: 1-36.

2. Lexell J, Taylor CC, Sjostrom M. What is the cause of the ageing atrophy? Total number, size and proportion of different fiber types studied in whole vastus lateralis muscle from 15- to 83-year-old men. J Neurol Sci 1988; 84: 275-294.

3. Yamauchi J, Mishima C, Nakayama S, Ishii N. Aging-related differences in maximum force, unloaded velocity and power of human leg multi-joint movement. Gerontology 2010; 56: 167-174

4. Lauretani F, Russo CR, Bandinelli S, Bartali B, Cavazzini C, Di lorio A, et al. Age-associated changes in skeletal muscles and their effect on mobility: an operational diagnosis of sarcopenia. J Appl Physiol 2003; 95: 1851-1860.

5. Exercise prescription for older adults with osteoarthritis pain: consensus practice recommendations. A supplement suggest that training also did not change the cardiovascular responses to the same aerobic stress.

Contrary to the hypothesis, the results of this study showed that ST and PT did not improve work efficiency during aerobic exercise, and did not decrease the cardiovascular responses to submaximal or maximal aerobic effort. The practical implication of these findings is that they did not support the recommendation of resistance exercises for decreasing cardiovascular load during aerobic tasks.

It is important to address some limitations of this study. Among them, no session for familiarization with the exercise test was held, which may explain the changes observed in the $\mathrm{C}$ group. However, the presence of the $\mathrm{C}$ group in the study design allowed us to control for this familiarization effect. Resistance training effects are mainly influenced by the training characteristics (38). Thus, caution should be taken when extrapolating the findings of the present study to other resistance training regimens. In addition, the volunteers included in the present study were healthy adults aged 60 to 80 years, and results might be different in subjects with other characteristics.

In conclusion, ST and PT did not decrease the metabolic cost of submaximal and maximal aerobic exercises, as had been previously suggested. As a consequence, they did not blunt the cardiovascular load increase during this kind of exercise.

\section{Acknowledgments}

The authors wish to thank the volunteers involved in this study. We also thank the students of the Neuromuscular Adaptation Laboratory for providing resistance training to the volunteers. Research supported by FAPESP (\#07/56653-1 and \#07/00788-6), CNPq (\#471600/2008-3), CAPES, and Head of the Psychopharmacology Incentive Fund Association. to the AGS Clinical Practice Guidelines on the management of chronic pain in older adults. J Am Geriatr Soc 2001; 49: 808-823.

6. American College of Sports Medicine. ACSM's guidelines for exercise testing and prescription. Philadelphia: Lippincott Williams \& Wilkins; 2010.

7. Nelson ME, Rejeski WJ, Blair SN, Duncan PW, Judge JO, King AC, et al. Physical activity and public health in older adults: recommendation from the American College of Sports Medicine and the American Heart Association. Circulation 2007; 116: 1094-1105.

8. Trappe S, Williamson D, Godard M. Maintenance of whole muscle strength and size following resistance training in older men. J Gerontol A Biol Sci Med Sci 2002; 57: B138B143.

9. Bottaro M, Machado SN, Nogueira W, Scales R, Veloso $\mathrm{J}$. Effect of high versus low-velocity resistance training on 
muscular fitness and functional performance in older men. Eur J Appl Physiol 2007; 99: 257-264.

10. Miszko TA, Cress ME, Slade JM, Covey CJ, Agrawal SK, Doerr CE. Effect of strength and power training on physical function in community-dwelling older adults. $J$ Gerontol $A$ Biol Sci Med Sci 2003; 58: 171-175.

11. Wood RH, Reyes R, Welsch MA, Favaloro-Sabatier J, Sabatier M, Matthew LC, et al. Concurrent cardiovascular and resistance training in healthy older adults. Med Sci Sports Exerc 2001; 33: 1751-1758.

12. Martel GF, Hurlbut DE, Lott ME, Lemmer JT, Ivey FM, Roth $\mathrm{SM}$, et al. Strength training normalizes resting blood pressure in 65- to 73-year-old men and women with high normal blood pressure. J Am Geriatr Soc 1999; 47: 1215-1221.

13. Simons $R$, Andel $R$. The effects of resistance training and walking on functional fitness in advanced old age. J Aging Health 2006; 18: 91-105.

14. Taaffe DR, Galvao DA, Sharman JE, Coombes JS. Reduced central blood pressure in older adults following progressive resistance training. J Hum Hypertens 2007; 21: 96-98.

15. Tsutsumi T, Don BM, Zaichkowsky LD, Delizonna LL. Physical fitness and psychological benefits of strength training in community dwelling older adults. Appl Human Sci 1997; 16: 257-266.

16. McCartney N, McKelvie RS, Martin J, Sale DG, MacDougall JD. Weight-training-induced attenuation of the circulatory response of older males to weight lifting. $J$ Appl Physiol 1993; 74: 1056-1060.

17. Filipovsky J, Ducimetiere P, Safar ME. Prognostic significance of exercise blood pressure and heart rate in middleaged men. Hypertension 1992; 20: 333-339.

18. Bortolotto LA, Hanon O, Franconi G, Boutouyrie P, Legrain $S$, Girerd X. The aging process modifies the distensibility of elastic but not muscular arteries. Hypertension 1999; 34: 889-892.

19. Laitinen T, Hartikainen J, Vanninen E, Niskanen L, Geelen G, Lansimies E. Age and gender dependency of baroreflex sensitivity in healthy subjects. J Appl Physiol 1998; 84: 576583.

20. Lakatta EG, Levy D. Arterial and cardiac aging: major shareholders in cardiovascular disease enterprises: Part II: the aging heart in health: links to heart disease. Circulation 2003; 107: 346-354.

21. O'Rourke MF, Hashimoto J. Mechanical factors in arterial aging: a clinical perspective. J Am Coll Cardiol 2007; 50: 1-13.

22. Parati G, Frattola A, Di Rienzo M, Castiglioni P, Mancia G. Broadband spectral analysis of blood pressure and heart rate variability in very elderly subjects. Hypertension 1997; 30: 803-808

23. Singh D, Vinod K, Saxena SC, Deepak KK. Spectral evaluation of aging effects on blood pressure and heart rate variations in healthy subjects. J Med Eng Technol 2006; 30: $145-150$

24. Ministério da Saúde. Painel de Indicadores do SUS. 2006. http://www.fss.org.br/arq_biblioteca/painel_indicadores_ do_sus.pdf. Accessed July 15, 2011.

25. O'Brien E, Asmar R, Beilin L, Imai Y, Mancia G, Mengden $\mathrm{T}$, et al. Practice guidelines of the European Society of Hypertension for clinic, ambulatory and self blood pressure measurement. J Hypertens 2005; 23: 697-701.

26. Brown L, Weir J. The American Society of Exercise (ASEP) procedures recommendation I: Accurate assessment of muscular strength and power. $J$ Exerc Physiologyonline 2001; 4: 1-21.

27. Brazilian Society of Cardiology. II Guidelines on ergometric tests of the Brazilian Society of Cardiology. Arq Bras Cardiol 2002; 78 (Suppl 2): S1-S17.

28. Henwood TR, Riek S, Taaffe DR. Strength versus muscle power-specific resistance training in community-dwelling older adults. J Gerontol A Biol Sci Med Sci 2008; 63: 8391.

29. McCartney N, Hicks AL, Martin J, Webber CE. Long-term resistance training in the elderly: effects on dynamic strength, exercise capacity, muscle, and bone. J Gerontol A Biol Sci Med Sci 1995; 50: B97-B104.

30. Anton MM, Cortez-Cooper MY, DeVan AE, Neidre DB, Cook $\mathrm{JN}$, Tanaka H. Resistance training increases basal limb blood flow and vascular conductance in aging humans. $J$ Appl Physiol 2006; 101: 1351-1355.

31. Castaneda C, Layne JE, Munoz-Orians L, Gordon PL, Walsmith J, Foldvari $\mathrm{M}$, et al. A randomized controlled trial of resistance exercise training to improve glycemic control in older adults with type 2 diabetes. Diabetes Care 2002; 25: 2335-2341.

32. Cononie CC, Graves JE, Pollock ML, Phillips MI, Sumners C, Hagberg JM. Effect of exercise training on blood pressure in 70- to 79-yr-old men and women. Med Sci Sports Exerc 1991; 23: 505-511.

33. Burstyn P, O'Donovan B, Charlton I. Blood pressure variability: the effects of repeated measurement. Postgrad Med J 1981; 57: 488-491.

34. McGrady A, Higgins JT Jr. Effect of repeated measurements of blood pressure on blood pressure in essential hypertension: role of anxiety. J Behav Med 1990; 13: 93-101.

35. Tonino RP, Driscoll PA. Reliability of maximal and submaximal parameters of treadmill testing for the measurement of physical training in older persons. J Gerontol 1988; 43: M101-M104.

36. Holviala J, Hakkinen A, Karavirta L, Nyman K, Izquierdo M, Gorostiaga EM, et al. Effects of combined strength and endurance training on treadmill load carrying walking performance in aging men. $J$ Strength Cond Res 2010; 24: 1584-1595

37. Hagerman FC, Walsh SJ, Staron RS, Hikida RS, Gilders RM, Murray TF, et al. Effects of high-intensity resistance training on untrained older men. I. Strength, cardiovascular, and metabolic responses. J Gerontol A Biol Sci Med Sci 2000; 55: B336-B346.

38. Kraemer WJ, Ratamess NA. Fundamentals of resistance training: progression and exercise prescription. Med Sci Sports Exerc 2004; 36: 674-688. 